\title{
Aves de fragmentos florestais em área de cultivo de cana-de-açúcar no sudeste do Brasil
}

\author{
Augusto Piratelli ${ }^{1}$, Viviane A. Andrade ${ }^{1,2} \&$ Mauri Lima Filho ${ }^{3}$
}

\author{
1. Departamento de Biologia Animal, Instituto de Biologia, Universidade Federal Rural do Rio de Janeiro (UFRRJ), Rodovia BR 465, Km \\ 07, 23890-000 Seropédica, RJ, Brasil. (pirateli@ufrrj.br) \\ 2. Programa de Pós-Graduação em Biologia Animal, IB, UFRRJ. (vivalves2@yahoo.com.br) \\ 3. Campus Dr. Leonel Miranda, UFRRJ, Estrada do Açúcar, Km 05, 28020-560 Campos dos Goytacazes, RJ. (clmufrrj@ rol.com.br)
}

\begin{abstract}
Birds of forest fragments in area of sugar-cane crops in southeastern Brazil. Birds of four forest fragments in areas of extensive sugar-cane plantation were studied in Campos dos Goytacazes, northern Rio de Janeiro State, Brazil, from October 2000 to July 2001. The basic diet of sampled species and their trophic guild allocation were determined. The study was carried out by means of capture with mist nets, visual and auditive records and analysis of faeces. Forty-four species were recorded and grouped in eight trophic guilds (insectivores, granivores, carnivores, frugivores, piscivores, nectarivores, omnivores and carrion eaters). The species were also allocated into smaller guilds, associated to their habitats. Some species only fly over the fragments, as Egretta thula (Molina, 1782), while others are residents, as Manacus manacus (Linnaeus, 1766), and some used these fragments for nocturnal resting, as Amazona amazonica (Linnaeus, 1766). Small understory species apparently do not travel between fragments, given their relatively large spacing. Raptors, such as Rupornis magnirostris (Gmelin, 1789) live in both fragments and nearby open areas. Fragments in critical situation, supporting mainly generalists and/or edge species, still support some species with ecological interest, such as Rhynchocyclus olivaceus (Temminck, 1820) and A. amazonica.
\end{abstract}

KEYWORDS. Birds, forest fragments, guilds, sugar-cane, Rio de Janeiro.

RESUMO. Foi estudada a avifauna de quatro fragmentos florestais em uma área de cultivo de cana-de-açúcar na região de Campos dos Goytacazes, norte do estado do Rio de Janeiro. A dieta básica e a estrutura das guildas tróficas foi determinada. O estudo foi realizado de outubro de 2000 a julho de 2001, utilizando-se capturas com redes ornitológicas, registros visuais e auditivos e análise de fezes. Quarenta e quatro espécies foram registradas e agrupadas em oito guildas tróficas (insetívoros, granívoros, carnívoros, frugívoros, piscívoros, nectarívoros, onívoros e detritívoros). Estas espécies foram também subdivididas em guildas mais específicas, associadas a seus hábitats. Algumas espécies apenas sobrevoaram os fragmentos, como Egretta thula (Molina, 1782), enquanto outras foram consideradas residentes, como Manacus manacus (Linnaeus, 1766). Algumas, como Amazona amazonica (Linnaeus, 1766), somente utilizaram os fragmentos para repouso noturno. Espécies pequenas de sub-bosque provavelmente não se deslocaram entre fragmentos, dada a relativa grande distância entre eles. Predadores como Rupornis magnirostris (Gmelin, 1789) utilizaram tanto os fragmentos quando as áreas abertas e canaviais em seu entorno. Estes fragmentos estão em situação crítica, abrigando principalmente espécies generalistas e/ou especialistas de bordas; porém ainda são utilizados de alguma forma por espécies de interesse ecológico, como Rhynchocyclus olivaceus (Temminck, 1820) e A. amazonica.

PALAVRAS-CHAVE. Aves, fragmentos, guildas, cana-de-açúcar, Rio de Janeiro.

As atividades humanas têm causado inúmeros impactos ambientais, reduzindo as áreas de vegetação nativa contínuas e transformando-as em fragmentos florestais isolados. Espécies como aves predadoras de topo de cadeia alimentar ou predadores/dispersores de sementes, que invariavelmente necessitam de milhares de hectares para sobreviverem, rapidamente são afetadas (TERBORGH, 1992).

São vários os efeitos da fragmentação sobre as comunidades de aves (BornscheIn \& REINERT, 2000). Bandos mistos podem ter sua estabilidade, riqueza, tamanho e composição alterados em decorrência deste processo e do tamanho das formações vegetais remanescentes (Maldonado-Coelho \& Marini, 2000). WiLLIS (1979) relatou que aves escaladoras de troncos e galhos (Picidae e Dendrocolaptidae) são as que mais rapidamente desaparecem. D'Angelo Neto et al. (1998) verificaram redução de cerca de $48 \%$ das espécies florestais em pequenos trechos de mata em Minas Gerais. Aves dependentes de mata tendem a desaparecer nos menores remanescentes, enquanto outras, como as granívoras, podem ser favorecidas com o isolamento dos fragmentos, pelo aumento da área de bordas, hábitats mais utilizados por estas últimas (ANJos, 1998). RESTREPO \& Gómez (1998) observaram os efeitos de borda sobre as diferentes guildas de alimentação no sudoeste da Colômbia. Concluíram que, além da distância, o tempo transcorrido desde a formação da borda e as estações do ano também afetam a abundância, de modo distinto, nas diferentes guildas.

Uma das monoculturas que têm substituído a Mata Atlântica em algumas regiões do Rio de Janeiro é a canade-açúcar. A maior parte da região norte do Estado atualmente é coberta por pastos e culturas de cana-deaçúcar, mandioca e arroz, restando como matas remanescentes mais expressivas as do Parque Estadual do Desengano, com 22.500 ha, e a Fazenda União, com 2.000 ha (PACHECO et al., 1996). As monoculturas de canade-açúcar são ambientes particularmente pobres em espécies de aves, mesmo quando comparados a outros ambientes alterados (Petit et al., 1999; Martin \& CATterall, 2001). 
O objetivo deste trabalho foi estudar a avifauna em quatro fragmentos florestais isolados no interior de canaviais na região norte do Estado do Rio de Janeiro, observando algumas características ecológicas das espécies e os possíveis efeitos da fragmentação florestal sobre elas.

\section{MATERIAL E MÉTODOS}

O município de Campos dos Goytacazes $\left(21^{\circ} 75^{\prime} \mathrm{S}\right.$, $41^{\circ} 32^{\prime}$ W) localiza-se na região norte do Estado do Rio de Janeiro, tendo uma área de $4.027 \mathrm{~km}^{2}$ e altitude de $13 \mathrm{~m}$ no distrito sede do município (IBGE, 2004). Foram amostrados quatro fragmentos localizados entre as plantações de cana-de-açúcar da Usina Santa Cruz (figs. 1-4). O fragmento $\mathrm{F} 1$ ( $\left.9,95 \mathrm{ha} ; 2^{\circ} 42^{\prime} \mathrm{S}, 4^{\circ}{ }^{\circ} 9^{\prime} \mathrm{W}\right)$ distava 825 m do fragmento F2 (18,24 ha; $\left.21^{\circ} 43^{\prime} \mathrm{S}, 41^{\circ} 29^{\prime} \mathrm{W}\right), 825$ m de F3 (27,28 ha; $\left.21^{\circ} 43^{\prime} \mathrm{S}, 41^{\circ} 28^{\prime} \mathrm{W}\right)$ e $1.375 \mathrm{~m}$ de F4 (4,00 ha; $\left.21^{\circ} 43^{\prime} \mathrm{S}, 41^{\circ} 28^{\prime} \mathrm{W}\right)$. O fragmento F2 distava 525 $\mathrm{m}$ do fragmento $\mathrm{F} 3$ e $1.425 \mathrm{~m}$ de F4. A distância entre os fragmentos F3 e F4 era de $850 \mathrm{~m}$.

As aves foram amostradas utilizando-se capturas com redes ornitológicas e registros visuais e auditivos. Para as capturas, utilizaram-se redes ornitológicas de malha $36 \mathrm{~mm}$, dispostas em transectos lineares (adaptado da metodologia de BIERREGAARD, 1990) em trilhas já existentes. As redes eram abertas ao alvorecer, em horários que variaram conforme as estações do ano, e fechadas no final do dia. As coletas iniciaram-se em outubro de 2000 e estenderam-se até junho de 2001, totalizando aproximadamente 1.000 horas-rede. Para registros visuais (com auxílio de binóculos 10x50) e auditivos, foram efetuadas caminhadas aleatórias ao longo das trilhas e ao redor dos fragmentos, além de percursos esporádicos ao longo dos canaviais, totalizando cerca de 50 horas de observação. Para a marcação das aves, foram utilizadas anilhas metálicas cedidas pelo CEMAVE/IBAMA. A nomenclatura e a ordem sistemática das espécies seguiram Sick (1997).

Para determinação da dieta, as aves capturadas foram mantidas por cerca de 10 minutos em sacos de pano contendo papel filtro em seu interior, para que se obtivessem amostras de suas fezes (Dekinga \& Piersma, 1993; TAYLOR \& O'HALLORAN, 1997). As fezes e regurgitos obtidos com este procedimento foram acondicionados em recipientes de plástico etiquetados e trazidos para o laboratório, onde foram triados com auxílio de microscópio estereoscópico e estiletes de ponta fina. Em relação àquelas espécies capturadas, das quais não se obtiveram amostras de sua dieta, foi considerado como hábito alimentar principal aquele mais amplamente descrito na literatura (WILLIS, 1979; Sick, 1997), além de observações no campo. Para comparar a composição trófica nos quatro fragmentos, foi utilizado o teste de Chiquadrado, empregando-se o pacote estatístico Statistix 8.0.

\section{RESULTADOS E DISCUSSÃO}

No total, foram amostradas 44 espécies, representantes de 23 famílias, e efetuadas 23 capturas (tab. I). Estas 44 espécies representam $10,6 \%$ das 414 conhecidas para a região norte fluminense que, segundo PACHECO et al. (1996), seriam em sua maioria representativas da baixada quente florestada, de vegetação pioneira litorânea, de
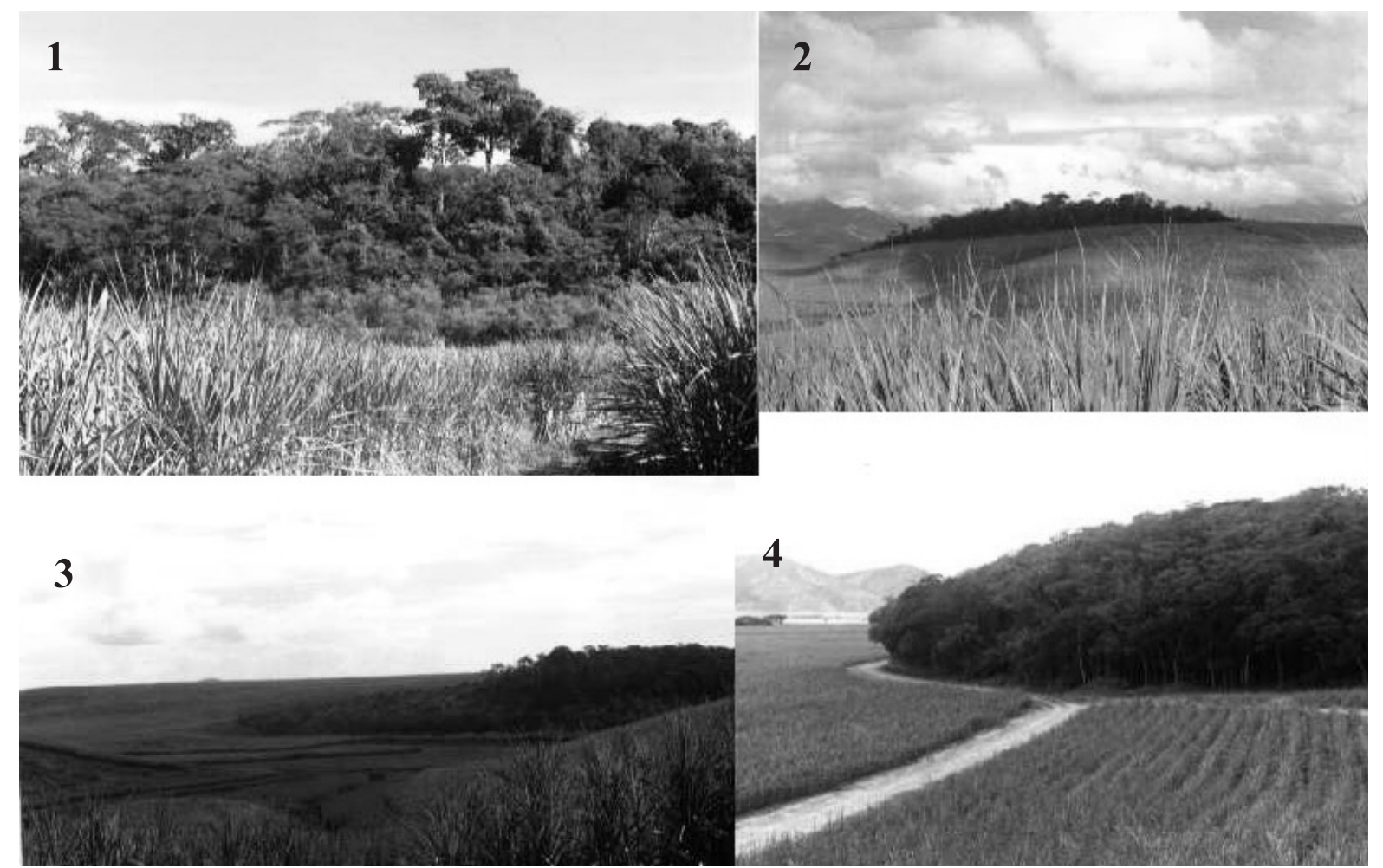

Figs. 1-4. Vista parcial dos quatro fragmentos amostrados na Usina Santa Cruz, Campos dos Goytacazes, RJ, entre outubro de 2000 e junho de 2001. 
áreas alagadas e principalmente das imensas áreas alteradas pela atividade humana.

A maioria das espécies $(n=21)$ foi registrada utilizando o interior e a borda dos fragmentos $(47,7 \%)$. Dezesseis espécies eram típicas de borda $(36,4 \%)$, seis $(13,6 \%)$ apenas sobrevoaram os remanescentes florestais (tendo uma delas pousado em seu interior) e Amazona amazonica (Linnaeus, 1766) (2,3\%) utilizou-os como abrigo noturno, partindo em pequenos bandos no começo da manhã e retornando ao final do dia. Entre as espécies capturadas, destacou-se Rhynchocyclus olivaceus (Temminck, 1820), que se encontra na lista das espécies ameaçadas de extinção no Estado do Rio de Janeiro, com status de vulnerável (Alves et al., 2000). Tais proporções mantiveram-se quase inalteradas nas quatro áreas, independentemente de seu tamanho, e nenhuma das espécies observadas pode ser considerada exclusiva de ambientes florestais; todas as encontradas no interior das matas também exploram suas periferias. Pequenas manchas florestais, em geral, têm menos espécies dependentes de floresta do que áreas maiores (AlEIXo \& VIELliard, 1995; MARINI, 2001). De acordo com LAURANCE et al. (2002), o efeito de borda, agindo na redução da abundância de aves de sub-bosque, pode atingir até 200 $\mathrm{m}$ em seu interior, de onde supõe-se que o reduzido tamanho dos remanescentes aqui estudados os torna grandes bordas, não suportando, de modo geral, espécies mais especializadas (fig. 5).

Várias possíveis conseqüências da severa degradação puderam ser detectadas, tais como as baixas

Tabela I. Espécies de aves amostradas, sua ocorrência e utilização dos fragmentos estudados (F1, F2, F3, F4) e guildas tróficas na região norte fluminense. Os algarismos indicam o número de indivíduos capturados para a espécie no local; " $\mathrm{x}$ " indica ocorrência da espécie, sem capturas (AN, abrigo noturno; B, borda; CAC, consumidores de artrópodes de chão no sub-bosque; CC, consumidores de carniça, necrófagas; $\mathrm{CD}$, carnívoros diurnos; CN, carnívoros noturnos; GB, granívoros de bordas e áreas abertas; GC, granívoros de chão; GD, granívoros de dossel; I, interior; IA, insetívoras aéreas; IB, insetívoros de borda; IBC, insetívoras de bambus ou cipós; IS, insetívoros de sub-bosque; IT, insetívoros de troncos e brotos; NE, nectarívoras; OF, onívoras ou frugívoras de bordas ou áreas abertas; OS, onívoras de sub-bosque; PI, piscívoras; S, sobrevôo).

\begin{tabular}{|c|c|c|c|c|c|c|}
\hline Espécies & F1 & $\mathrm{F} 2$ & F3 & F4 & Uso dos fragmentos & Guilda trófica \\
\hline Crypturellus tataupa (Temminck, 1815) & & $\mathrm{x}$ & $\mathrm{x}$ & & $\mathrm{I}$ & $\mathrm{GC}$ \\
\hline Casmerodius albus (Linnaeus, 1758) & & & & $\mathrm{x}$ & $\mathrm{S}$ & PI \\
\hline Egretta thula (Molina, 1758) & & $\mathrm{x}$ & & $\mathrm{x}$ & $\mathrm{S}$ & PI \\
\hline Cathartes aura Linnaeus, 1758 & & & & $\mathrm{x}$ & $\mathrm{S}$ & $\mathrm{CC}$ \\
\hline Coragyps atratus (Bechstein, 1758) & $\mathrm{x}$ & $\mathrm{x}$ & & & $\mathrm{S}$ & $\mathrm{CC}$ \\
\hline Poliborus plancus (Miller, 1777) & $\mathrm{x}$ & $\mathrm{x}$ & $\mathrm{x}$ & $\mathrm{x}$ & B & $\mathrm{CD}$ \\
\hline Rupornis magnirostris (Gmelin, 1789) & $\mathrm{x}$ & $\mathrm{x}$ & & $\mathrm{x}$ & $\mathrm{S}$ e I & $\mathrm{CD}$ \\
\hline Herpetotheres cachinnans Linnaeus, 1758 & $\mathrm{x}$ & & & & B & $\mathrm{CD}$ \\
\hline Milvago chimachima (Vieillot, 1816) & & & & $\mathrm{x}$ & B & $\mathrm{CD}$ \\
\hline Aramides cajanea (Müller, 1776) & & & $\mathrm{x}$ & & B & $\mathrm{CAC}$ \\
\hline Porzana albicollis (Vieillot, 1819) & & $\mathrm{x}$ & & & B & CAC \\
\hline Cariama cristata (Linnaeus, 1766) & $\mathrm{x}$ & $\mathrm{x}$ & & & B & $\mathrm{CD}$ \\
\hline Columba cayennensis Bonnaterre, 1792 & & $\mathrm{x}$ & & & I & GB \\
\hline Columbina minuta (Linnaeus, 1766) & & $\mathrm{x}$ & $\mathrm{x}$ & & B & GB \\
\hline Columbina talpacoti (Temminck, 1811) & $\mathrm{x}$ & & & & B & GB \\
\hline Amazona amazonica (Linnaeus, 1766) & $\mathrm{x}$ & $\mathrm{x}$ & $\mathrm{x}$ & $\mathrm{x}$ & AN & GD \\
\hline Crotophaga ani Linnaeus, 1758 & $\mathrm{x}$ & $\mathrm{x}$ & $\mathrm{x}$ & & $\mathrm{B}$ & IB \\
\hline Tyto alba (Scopoli, 1769) & $\mathrm{x}$ & & & & I & $\mathrm{CN}$ \\
\hline Speotyto cunicularia (Molina, 1782) & & & & $\mathrm{x}$ & B & $\mathrm{CN}$ \\
\hline Nyctidromus albicollis (Gmelin, 1789) & $\mathrm{x}$ & $\mathrm{x}$ & $\mathrm{x}$ & & I & IB \\
\hline Hydropsalis brasiliana (Gmelin, 1789) & & & 1 & & I & IB \\
\hline Thalurania glaucopis (Gmelin, 1788) & & & & $\mathrm{x}$ & I & $\mathrm{NE}$ \\
\hline Amazilia fimbriata (Gmelin, 1788) & & & $\mathrm{x}$ & $\mathrm{x}$ & I & NE \\
\hline Piccumnus cirratus Temminck, 1825 & & $\mathrm{x}$ & 1 & $\mathrm{x}$ & I & I T \\
\hline Colaptes campestris (Vieillot, 1818) & $\mathrm{x}$ & $\mathrm{x}$ & $\mathrm{x}$ & $\mathrm{x}$ & B & OF \\
\hline Camptostoma obsoletum (Temminck, 1824) & & & $\mathrm{x}$ & & I & OS \\
\hline Elaenia flavogaster (Thunberg, 1822) & $\mathrm{x}$ & $\mathrm{x}$ & & & I & $\mathrm{OF}$ \\
\hline Serpophaga subcristata (Vieillot, 1817) & & & $\mathrm{x}$ & & I & IB \\
\hline Myiornis auricularis (Vieillot,1818) & & 3 & 1 & 1 & I & IBC \\
\hline Rhynchocyclus olivaceus (Temminck, 1820) & $\mathrm{x}$ & & 4 & 2 & I & IS \\
\hline Myiarchus tyrannulus (Müller, 1776) & & 1 & 1 & & I & IB \\
\hline Pitangus sulphuratus (Linnaeus, 1776) & $\mathrm{x}$ & & & & I e B & OF \\
\hline Tyrannus melancholicus Vieillot, 1819 & & $\mathrm{x}$ & & & B & IB \\
\hline Pachyramphus polychopterus (Vieillot, 1818) & $\mathrm{x}$ & $\mathrm{x}$ & & & I & IS \\
\hline Manacus manacus (Linnaeus, 1766) & 3 & 1 & & $\mathrm{x}$ & I & OS \\
\hline Notiochelidon cyanoleuca (Vieillot, 1817) & $\mathrm{x}$ & & & & $\mathrm{S}$ & IA \\
\hline Troglodytes aedon Vieillot, 1807 & $\mathrm{x}$ & $\mathrm{x}$ & 3 & $\mathrm{x}$ & B & IB \\
\hline Hylophilus poicilotis Temminck, 1822 & $\mathrm{x}$ & & & & I & IB \\
\hline Tachyphonus coronatus (Vieillot, 1822) & & & $\mathrm{x}$ & & I & $\mathrm{OF}$ \\
\hline Euphonia chlorotica (Linnaeus, 1776) & $\mathrm{x}$ & $\mathrm{x}$ & & & I & OS \\
\hline Tangara cayana (Linnaeus, 1776) & $\mathrm{x}$ & & & 1 & I & $\mathrm{OF}$ \\
\hline Volatina jacarina Linnaeus, 1776 & & & $\mathrm{x}$ & & B & GB \\
\hline Sporophila caerulescens (Vieillot, 1817) & & & $\mathrm{x}$ & & B & GB \\
\hline Estrilda astrild (Linnaeus, 1758) & $\mathrm{x}$ & & & & B & GB \\
\hline Total & 22 & 22 & 20 & 17 & & \\
\hline
\end{tabular}


taxas de captura, baixa diversidade de espécies, grande número de espécies generalistas e/ou típicas de áreas abertas, além da ausência de espécies tipicamente dependentes de ambientes florestais (SILVA, 1995). PetiT et al. (1999) e MARTIN \& CATTERALl (2001) argumentam que monoculturas como a cana-de-açúcar podem produzir grandes impactos sobre a avifauna, já que diminuem a complexidade estrutural da vegetação, reduzindo também a disponibilidade de recursos alimentares, principalmente para aves frugívoras.

Além do reduzido tamanho, as distâncias entre os remanescentes também podem ter contribuído para a baixa riqueza de espécies encontrada no presente estudo. Em razão da distância, apenas aquelas espécies mais associadas às áreas abertas ou espécies de maior porte poderiam transitar entre os fragmentos. PoulsEN (1994) concluiu que a maioria das espécies que estudou transitava entre manchas de vegetação distantes até $80 \mathrm{~m}$. Anjos (1998) verificou que, além do tamanho, o nível de isolamento (distância) entre áreas de mata nativa também é importante na determinação da composição avifaunística, tendo verificado ainda que um fragmento de 7 ha apresentava um número de espécies semelhante a outro de 60 ha mais isolado.

O que pode determinar a presença de uma espécie em um ambiente degradado é sua mobilidade e sua tolerância à deterioração do hábitat (LENs et al., 2002). Entretanto, a mobilidade pode variar em uma mesma espécie, dependendo se os indivíduos estão solitários ou agregados a bandos, tornando-se mais móveis neste último caso (Poulsen, 1994). Laurance et al. (2002) constataram que, eventualmente, alguns Passeriformes amazônicos poderiam atravessar áreas abertas de até 320 m dependendo de sua motivação, mas que, em geral, para algumas espécies insetívoras, uma clareira de apenas 80 $\mathrm{m}$ pode ser uma barreira instransponível.
Foram encontrados representantes de oito guildas tróficas, havendo predomínio de espécies insetívoras $(\mathrm{n}=$ $15 ; 34,1 \%)$ e granívoras $(n=8 ; 18,2 \%)$ (tab. II), que estiveram representadas em todos os locais estudados. Não houve diferença significativa entre as áreas estudadas quanto à composição trófica $\left(\chi^{2}=19,14\right.$; n.s.). Nestas guildas, predominaram espécies insetívoras de bordas $(18,2 \%)$ e granívoras de bordas e áreas abertas (13,3\%) (tab. II).

MotTA-JÚNIOR (1990) relatou que em ambientes com altos índices de degradação ambiental há um número crescente de aves onívoras e, possivelmente, insetívoras menos especializadas, sucedendo o contrário no caso de frugívoras e insetívoras mais ou menos especializadas. Os resultados deste trabalho confirmam esses relatos, já que a maior parte das guildas tróficas foi composta por espécies de bordas ou áreas abertas, e com pouca ou nenhuma especialização quanto à sua dieta. $\mathrm{O}$ reduzido número de espécies frugívoras seria mais uma evidência das condições de degradação ambiental da área em questão.

Poulsen (1994) constatou, no Equador, que espécies frugívoras movem-se mais freqüentemente entre manchas de florestas do que insetívoras e onívoras. Frugívoras menos especializadas como Manacus manacus (Linnaeus, 1766) podem ser comumente encontradas em áreas alteradas, sofrendo menos os efeitos da fragmentação (Aleixo \& ViELliard, 1995).

De modo semelhante, espécies de borda também se deslocam com mais frequiência por entre mosaicos de vegetação do que as de interior de mata. MARINI (2001) observou em área de cerrado que as manchas florestais menores apresentaram número de espécies menor, porém sem alterações nas guildas tróficas, exceto as granívoras, que aumentaram em diversidade conforme a redução no tamanho da área.

Apesar de alterados, os fragmentos estudados

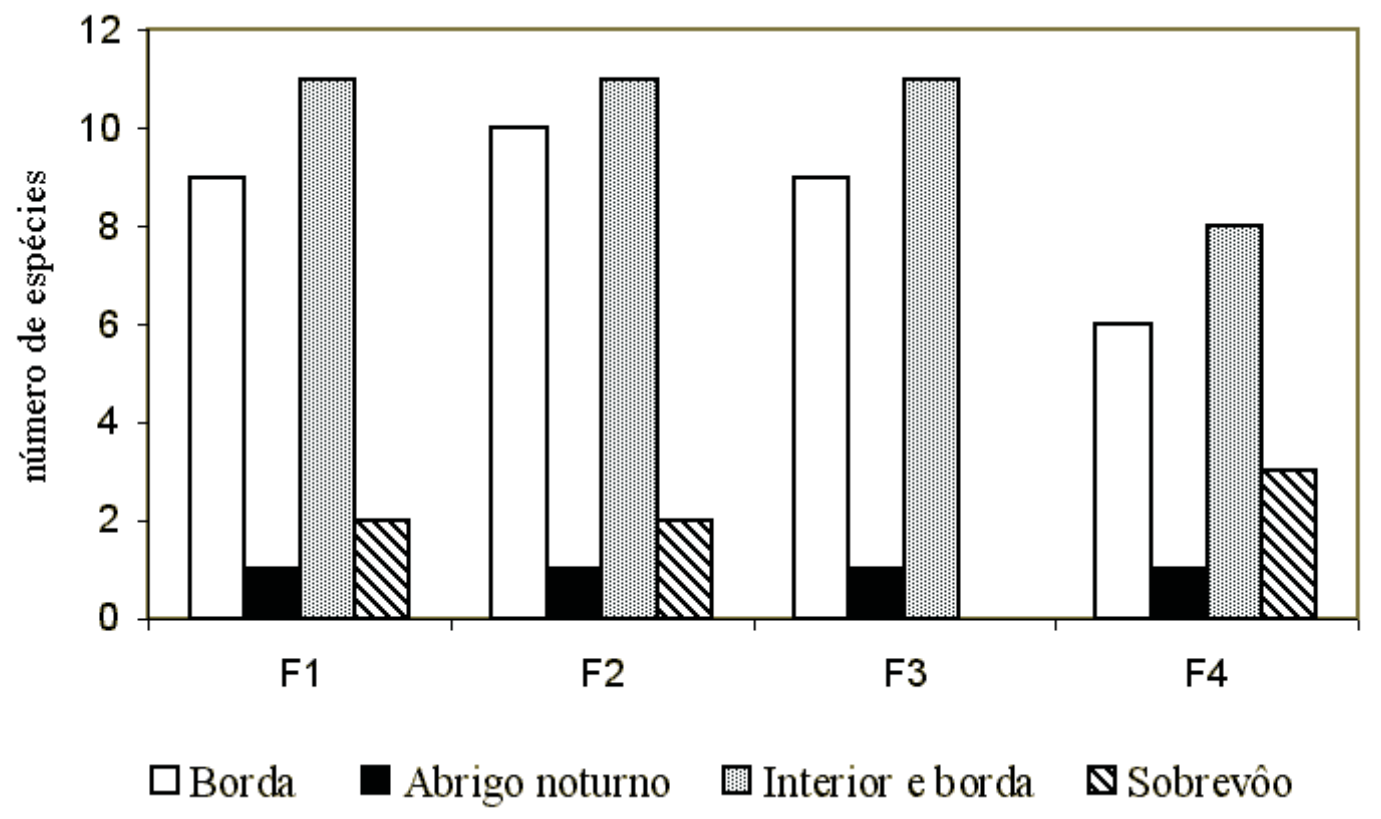

Fig. 5. Número de espécies de aves, amostradas de outubro de 2000 até junho de 2001, por tipo de utilização de hábitats em quatro fragmentos florestais (F1 a F4) na região de Campos dos Goytacazes (RJ). 
Tabela II. Número de espécies de aves, por guildas tróficas, encontrado em remanescentes florestais (F1, F2, F3, F4) na região de Campos dos Goytacazes (RJ), entre outubro de 2000 e junho de 2001. ( $\chi^{2}=19,14$; não significativo).

\begin{tabular}{|c|c|c|c|c|c|c|c|}
\hline Guildas & $\mathrm{F} 1$ & $\mathrm{~F} 2$ & F3 & F4 & $\mathrm{N}^{\circ}$ de ocorrências & Total de Espécies & Freqüência (\%) \\
\hline Carnívoros & 5 & 3 & 1 & 4 & 13 & 7 & 15,90 \\
\hline Necrófagos & 1 & 1 & 0 & 1 & 3 & 2 & 4,55 \\
\hline Frugívoros & 1 & 0 & 1 & 1 & 3 & 2 & 4,55 \\
\hline Granívoros & 3 & 4 & 5 & 1 & 13 & 8 & 18,20 \\
\hline Insetívoros & 7 & 9 & 10 & 4 & 30 & 15 & 34,10 \\
\hline Nectarívoros & 0 & 0 & 1 & 2 & 3 & 2 & 4,55 \\
\hline Onívoros & 5 & 4 & 2 & 2 & 13 & 6 & 13,60 \\
\hline Piscívoros & 0 & 1 & 0 & 2 & 3 & 2 & 4,55 \\
\hline Total & 22 & 22 & 20 & 17 & & 44 & 100,00 \\
\hline
\end{tabular}

ainda devem ser mantidos e até mesmo recuperados, pois espécies generalistas usuárias de áreas abertas também podem usufruir de florestas como abrigo noturno e/ou para a nidificação (ANJos \& Boçon, 1999), aproveitandose por exemplo de ocos em árvores (BERG, 1997), recursos que não podem ser encontrados em ambientes como canaviais. Amazona amazonica foi uma espécie que tipicamente utilizou as áreas de mata para abrigo noturno, principalmente pela presença de espécies arbóreas. Chiarello (2000) observou situação semelhante para Cairina moschata (Linnaeus, 1758) em uma mata de 150 ha cercada por cana-de-açúcar no interior do Estado de São Paulo.

Faltam, na área da Usina Santa Cruz, espécies especialistas como vários predadores de topo de cadeia (Accipitridae), médios e grandes frugívoros (Tinamidae, Trogonidae, Ramphastidae), insetívoros de chão (certos Formicariidae), grandes insetívoros de troncos (Picidae e Dendrocolaptidae) e diversos frugívoros especializados (certos Pipridae e Thraupinae), o que pode ser considerado uma consequiência típica da redução dos ambientes florestais (D'Angelo Neto et al., 1998). A recuperação das matas atrairia tais espécies, transformando as áreas não somente em abrigos de espécies generalistas, mas também em hábitat de aves mais especializadas com ocorrência conhecida para a região.

Agradecimentos. A Ana B. F. Bacelar e Carlos E. S. Garske, pelo auxílio na coleta de dados; ao Dr. Mauri Manhães e demais colegas do "Campus Dr. Leonel Miranda" da Universidade Rural, pelo apoio logístico; à Usina Santa Cruz, por permitir os estudos em áreas de sua propriedade, ao CNPq, pela bolsa de Iniciação Científica concedida à segunda autora durante as coletas de dados.

\section{REFERÊNCIASBIBLIOGRÁFICAS}

Aleixo, A. \& Vielliard, J. M. E. 1995. Composição e dinâmica da avifauna da Mata de Santa Genebra, Campinas, SP, Brasil. Revista Brasileira de Zoologia 12(4):493-511.

Alves, M. A. S.; Pacheco, J. F.; Gonzaga, L. A. P; Cavalcanti, R. B.; Raposo, M.; Yamashita, C.; Maciel, N. C. \& Castanheira, M. 2000. Aves. In: Bergallo, H. G.; Duarte da Rocha, C. F.; Alves, M. A. S. \& VAN SluYs, M. orgs. A fauna ameaçada de extinção do Estado do Rio de Janeiro. Rio de Janeiro, Editora UERJ. cap. 9, p.113-124.

AnJos, L. 1998. Consequiências biológicas da fragmentação no norte do Paraná. Série Técnica do Instituto de Pesquisas e Estudos Florestais 12(32):87-94.

Anjos, L. \& Boçon, R. 1999. Bird communities in natural forest patches in southern Brazil. Wilson Bulletin 11(3):397-414.

BERG, A. 1997. Diversity and abundance of birds in relation to forest fragmentation, habitat quality and heterogeneity. Bird Study 44:355-366.

BierRegaARD, R. O., JR. 1990. Species composition and trophic organization of the understory bird community in a central Amazonian terra firme forest. In: Gentry, A. org. Four Neotropical rainforests. New Haven, Yale University. p.217-235.

Bornschein, M. R. \& Reinert, B. L. 2000. Aves de três remanescentes florestais do norte do estado do Paraná. Revista Brasileira de Zoologia 17(3):615-636.

Chiarello, A. G. 2000. Conservation value of a native forest fragment in a region of extensive agriculture. Revista Brasileira de Biologia 60(2):237-247.

D’Angelo Neto, S.; Venturin, N.; Oliveira-filho, T. A. \& Costa, F. A. F. 1998. Avifauna de quatro fisionomias florestais de pequeno tamanho (5-8 ha) no campus da UFLA. Revista Brasileira de Biologia 58(3):463-472.

Dekinga, A. \& Piersma, T. 1993. Reconstructing diet-composition on the basis of faeces in a mollusc-eating-wader, the knot Calidris canutus. Bird Study 40:144-156.

IBGE. 2004. Instituto Brasileiro de Geografia e Estatística, Cidades. Disponível em: 〈http://www1.ibge.gov.br〉. Acesso em: 09.02.2004

Laurance, W. F.; Lovejoy, T. E.; Vasconcelos, H. L.; Bruna, E. M.; Didham, R. K.; Stouffer, P. C.; Gascon, C.; Bierregaard, R. O.; Laurance, S. G. \& Sampaio, E. 2002. Ecosystem decay of Amazonian Forest fragments: a 22 -year investigation. Conservation Biology 16(3):605-618.

Lens, L.; Van Dongen, S.; Norris, K.; Githiru, M. \& Matthysen, E. 2002. Avian persistence in fragmented rain forest. Science 298: $1236-1238$

Maldonado-Coelho, M. \& Marini, M. Â. 2000. Effects of forest fragment size and successional stage on mixed-species bird flocks in southeastern Brazil. Condor 102:585-594.

MarinI, M. Â. 2001. Effects of forest fragmentation on birds of the cerrado region, Brazil. Bird Conservation International 11:11-23

Martin, T. G. \& Catterall, C. P. 2001. Do fragmented coastal heathlands have habitat value to birds in eastern Australia? Wildlife Research 28(1):17-31.

MotTA-JúnIOR, J. C. 1990. Estrutura trófica e composição de três hábitats terrestres na região central do Estado de São Paulo. Ararajuba 1:65-71.

Pacheco, J. F.; Parrini, R.; Fonseca, P. S. M.; Whitney, B. M. \& Maciel, N. C. 1996. Novos registros de aves para o Estado do Rio de Janeiro: Região Norte. Atualidades Ornitológicas 72:10-12.

Petit, L. J; Petit, D. R.; Christian, D. G. \& Powell, H. D. W. 1999. Bird communities of natural and modified habitats in Panama. Ecography 22(3):292-304.

Poulsen, B. O. 1994. Movements of single birds and mixedspecies flocks between isolated fragments of cloud forest in Ecuador. Studies on Neotropical Fauna and Environment 29(3): 149-160.

Restrepo, C. \& Gómez, N. 1998. Responses of understory birds to anthropogenic edges in a neotropical montane forest. Ecological Applications 8(1):170-183.

SICK, H. 1997. Ornitologia Brasileira. São Paulo, Nova Fronteira. $862 \mathrm{p}$. 
Silva, J. M. C. 1995. Birds of the Cerrado region, South America. Steenstrupia 21:69-92.

TAYlor, A. J. \& O'Halloran, J. 1997. The diet of the dipper Cinclus cinclus as represented by faecal and regurgitate pellets: a comparison. Bird Study 44:338-347.
Terborgh, J. 1992. Maintenance of diversity in tropical forests. Biotropica 24(2b):283-292.

WiLlis, E. O. 1979. The composition of avian communities in remanescent woodlots in southern Brazil. Papéis Avulsos de Zoologia 33(1):1-25.

Recebido em junho de 2003. Aceito em junho de 2005. ISSN 0073-4721 Artigo disponível em: www.scielo.br/isz 